\title{
EXCHANGE PF-RINGS AND ALMOST PP-RINGS
}

\author{
H. AL-EZEH \\ Department of Mathematics \\ University of Jordan \\ Amman, Jordan
}

(Received June 1, 1988 and in revised form August 11, 1988)

ABSTRACT. Let $R$ be a commutative ring with unity. In this paper, we prove that $R$ is an almost PP-PM-ring if and on $1 y$ if $R$ is an exchange PF-ring. Let $X$ be a completely regular Hausdorff space, and let $\beta X$ be the Stone Cech compactification of $X$. Then we prove that the $r$ ing $C(X)$ of all continuous real valued functions on $X$ is an almost $P P-$ ring if and only if $X$ is an F-space that has an open basis of clopen sets. Finally, we deduce that the ring $C(X)$ is an almost PP-ring if and only if $C(X)$ is a U-ring, i.e. for each $f \in C(X)$, there exists a unit $u \in C(X)$ such that $f=u|f|$.

KEY WORDS AND PHRASES. PF-ring, PP-ring, PM-ring, almost PP-ring, pure ideal, exchange ring, idempotents, Stone-Cech compactification, Boolean space and the ring of all continuous real valued functions over a space $X, C(X)$. 1980 AMS SUBJECT CLASSIFICATION CODES. Primary 13C13, Secondary 54 C40.

1. INTRODUCTION.

Al 1 rings considered in this paper are commutative with unity. Recall that $R$ is called a PF-ring if every principal ideal aR is a flat $R$-module, and it is called a $P R$-ring if every principal ideal aR is a projective R-module. An ideal $I$ of a $r i n g R$ is called pure if for each $x \in I$, there exists $y \in I$ such that $x y=x$. It is wel1-known that $R$ is a PF-ring if and only if for a $\in R$, annihilator ideal, ann(a), is pure, see Al-Ezeh [1]. Also it is well-known that $R$ is a PP-ring if for each $a \in R, a n n(a)$

is generated by an idempotent. In an earlier paper we introduced almost PP-rings as a generalization of PP-rings. A ring $R$ is called an almost PP-ring if for each $a \in R$, ann(a) is generated by idempotents of $R$. In fact, one can easily show that $R$ is an $R$ almost PP-ring if and only if for each $a \in R$ and $b \in$ ann(a), there exists an idempotent e in $\operatorname{ann}(a)$ such that be $=b$.

$R$

A ring $R$ is called an exchange ring if every element in $R$ can be written as the sum of $a$ unit and an idempotent. Exchange rings have been studied extensively, see for example Monk [2] and Johnstone [3]. Our aim in this paper is to study the 
relationship between exchange PF-rings and almost PP-rings. To carry out our study we need two more definitions. A ring $R$ is called a PM-ring if every proper prime ideal of $R$ is contained in a unlque maximal ideal of $R$. it is wel1-known that the ring of all continuous real valued functions over a completely regular Hausdorff space $X$, $C(X)$, is a PM-ring, see Gillman and Jerison [4]. A compact Hausdorff and totally disconnected space is called a Boolean (or Stone) space.

\section{MAIN RESULTS.}

First, we state a theorem that was proved by Johnstone [3] .

THEOREM 2.1 A ring $R$ is an exchange $r$ ing if and only $1 f$ it is a PM-ring and the space of maximal ideals of $R, \operatorname{Max}(R)$, is a Boolean space.

THEOREM 2.2 Let $R$ be an exchange PF-ring. Then it is an almost PP. PM-ring.

PROOF. Let $R$ be an exchange PF-ring. Let $a \in R$, and let $b \in$ ann(a). Since $R$ is $a$ PF-ring, there exists $c \in \operatorname{ann}(a)$ such that $b c=b$. Because $R$ is an exchange ring, $c=e+u$, where $e^{2}=e$ and $u$ is $a$ unit in $R$. Hence $c u^{-1}=e u^{-1}+1$, and so $\therefore-e=c u^{-1}(1-e)$. Since $a c=0, a(1-e)=0$. But $b c=b$, so $b(1-e)=u b$ since $c$ $=\mathrm{e}+\mathrm{u}$. Therefore $\mathrm{bu}^{-1}(1-\mathrm{e})=\mathrm{b}$. Consequently, $b(1-e)=b c u^{-1}(1-e)=b u^{-1}(1-e)=b$. Since $1-e \in \operatorname{ann}(a)$, $R$ is an almost PP-ring. By Theorem $1, R$ is a PM-ring. Hence $R$ is an almost PP-PM-ring.

Now we want to establish the converse of theorem 2.2. Clearly, every almost PPring is a PF-ring. So, by theorem 2.1, it is enough to show that the space of maximal ideals of $R, \operatorname{Max}(R)$, is a Boolean space. De Marco and Orsatt1 [5] proved that if $R$ is a PM-ring, then $\operatorname{Max}(R)$ is a compact Hausdorff space. So it is left to show that for an almost PP-PM-ring $R, \operatorname{Max}(R)$ is totally separated. That is for any two distinct maximal ideals $M$ and $M_{1}$ there exists a clopen set in $M a x(R)$ containing $M$ but not $M_{1}$.

THEOREM 2.3 Let $R$ be an almost PP-PM-ring. Then $R$ is an exchange PF-ring.

PROOF. By the above arguement, $R$ is a PF-PM-ring. Moreover, Max(R) is a compact Hausdorff space. Let $M_{1}, M_{2} \in \operatorname{Max}(R)$ and $M_{1} \neq M_{2}$. Since $R$ is a PM-ring, there exist $a \in M_{1}$ and $b \in M_{2}$ such that $a b=0$, see Contessa [6]. Because $R$ is an almost PPring, there exists an idempotent $e \underset{R}{\operatorname{ann}(b)}$ such that ea=a. Therefore e $M_{1}$ and $e \in M_{2}$. Since $e$ is an idempotent, $U=D(e)=\{M \in M a x(R): e \in M\}$ is a clopen set in $\operatorname{Max}(R)$ containing $M_{1}$ but not $M_{2}$. So, by theorem $2.1, R$ is an exchange PF-ring.

For a completely regular Hausdorff space $X$, the $r i n g$ of all continuous real valued functions, $C(X)$, is a PM-ring, see Gillman and Jerison [4]. Moreover, $\operatorname{Max}(C(X))$, is homeomorphic to $B X$, the St one-Cech compatification of $X$. Therefore $C(X)$ is an almost PP-ring if and only if $R$ is an exchange PF-ring. Consequently, $C(X)$ is an almost PP-ring if and only if it is a PF-ring and $6 X$ is a Boolean space. Al-Ezeh et al [7], proved that $C(X)$ is a PF-ring if and only if $X$ is an F-space, where $X$ is called an F-space if every finitely generated ideal is principal. It is wel1-known that $X$ is an F-space if and only if any two nonempty disjoint cozero sets are 
completely separated. Therefore, the ring $C(X)$ is an almost PP-ring if and only if $X$ is an F-space and $\beta X$ is a Boolean space. In fact, $B X$ is a Boolean space if and only if $X$ has an open basis of clopen sets. Thus the ring $C(X)$ is an almost $P P-r i n g$ if and only if $X$ is an $F$-space that has an open basis of clopen sets.

Finally, Gillman and Henriksen [8] defined the ring $C(X)$ to be a U-ring if for every $f \in C(X)$, there exists a unit $u \in C(X)$ such that $f=u|f|$. In the same paper they proved that the ring $\mathrm{C}(\mathrm{X})$ is a U-ring if and only if $X$ is an $F$-space and $\beta X$ is a Boolean space. So we get the following theorem.

THEOREM 2.4 The ring $C(X)$ is an almost PP-ring if and only if it is a U-ring.

We end this paper by giving some examples illustrating the relationships discussed above.

EXAMPLES.

1) Let $\mathrm{N}$ be the set of positive integers with the discrete topology. Let $B \mathrm{~N}$ be its Stone-Cech compactification. The space BNIN is a compact F-space, see Gillman and Jerisen [4]. Moreover, $\beta N I N$ is totally disconnected. Hence, the space BNIN is Boolean. So the ring $C(B N \backslash N)$ is an almost PP-ring. However, it is not a PP-ring because the space aNIN is not basically disconnected, see Brookshear [9].

2) Let $\mathrm{R}^{+}$be set of nonnegative reals endowed with the usual topology . The space $\beta R^{+} \backslash R^{+}$is a compact, connected F-space, see Gillman and Henriksen [8]. Thus, the ring $C(X)$ has no nontrivial idempotents. So, if it were an almost PP-ring, it would be an integral domain which is not the case because it has plenty of zero divisors. Consequently, $C\left(B R^{+} \backslash R^{+}\right)$is a $P F-r i n g s$ that is not an almost PP-ring.

3) The ring of integers is an almost PP-ring that is not a PM-ring, and so not an exchange ring.

\section{REFERENCES}

1. AL-EZEH, H. Some properties of polynomial rings. Internat. J. Math. and Math. Sci. 10(1987) 311-314.

2. MONK, G. A characterization of exchange rings. Proc. Amer. Math. Soc. 35(1972) 349-353.

3. JOHnStONE, P. "Stone Spaces", Cambridge Studies in Advanced Mathematics No. 3, Cambridge University Press, Cambridge (1982).

4. GILLMAN, L. and JERISON, M. "Rings of continuous Functions" Graduate Texts in Math. Vol. 43, Springer-Verlag, Berlin (1976).

5. DE MARCO, G. and ORSATTI, A. Commutative rings in which every prime ideal is contained in a maximal ideal. Proc. Amer. Math. Soc. 30(1979) 459-466.

6. CONTESSA, M. On PM-rings. Communications in algebra, 10(1982) 93-108.

7. AL-EZEH, H., NATSHEH, M. and HUSSEIN, D. Some properties of the ring of continuous functions. Arch der Math to appear (1988).

8. GILLMAN, L. and HENRIKSEN, M. Rings of continuous functions in which every finitely generated ideal is principal. Trans. Amer. Math. Soc. $82(1956)$, 366-391.

9. BROOKSHEAR, J. Projective ideals in the ring of continuous functions, Pacific of J. Math. $71(1977)$ 313-333. 


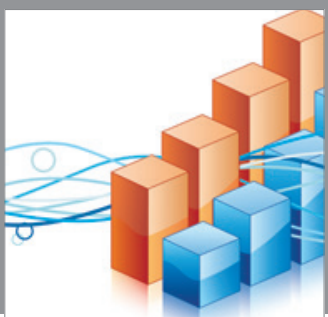

Advances in

Operations Research

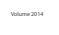

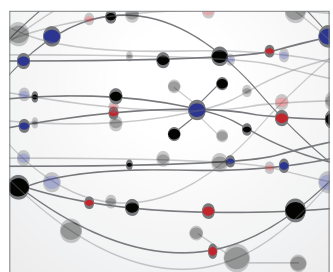

\section{The Scientific} World Journal
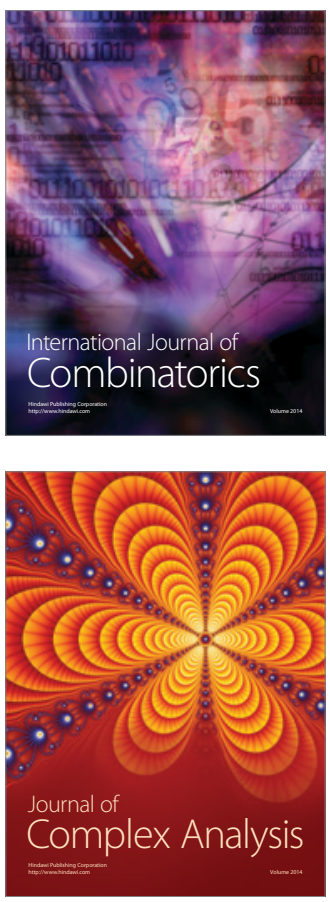

International Journal of

Mathematics and

Mathematical

Sciences
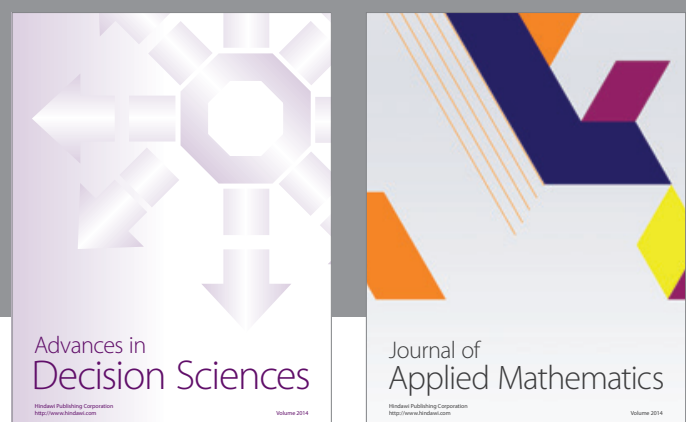

Journal of

Applied Mathematics
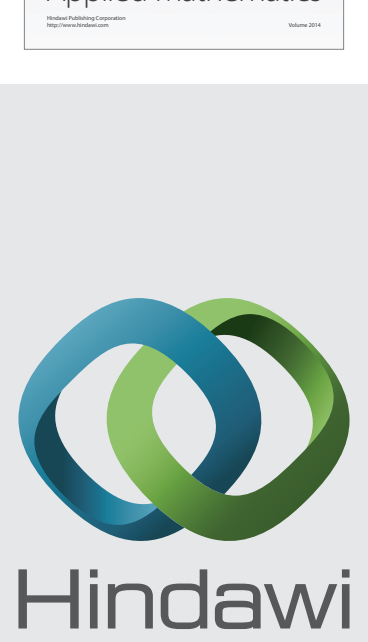

Submit your manuscripts at http://www.hindawi.com
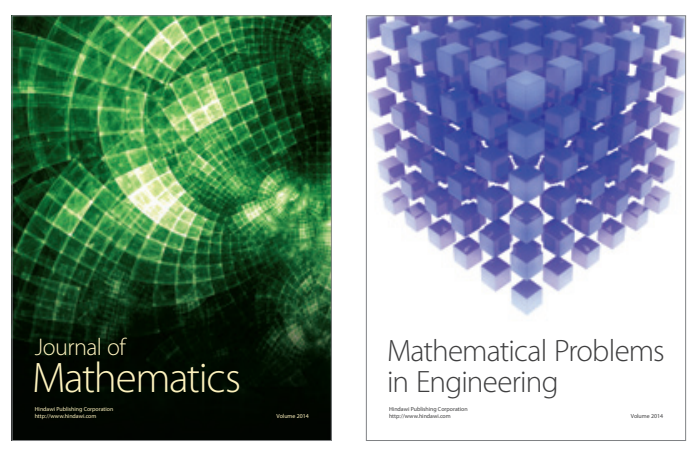

Mathematical Problems in Engineering
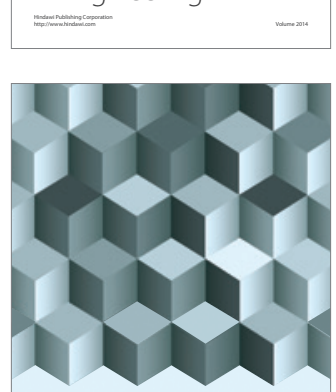

Journal of

Function Spaces
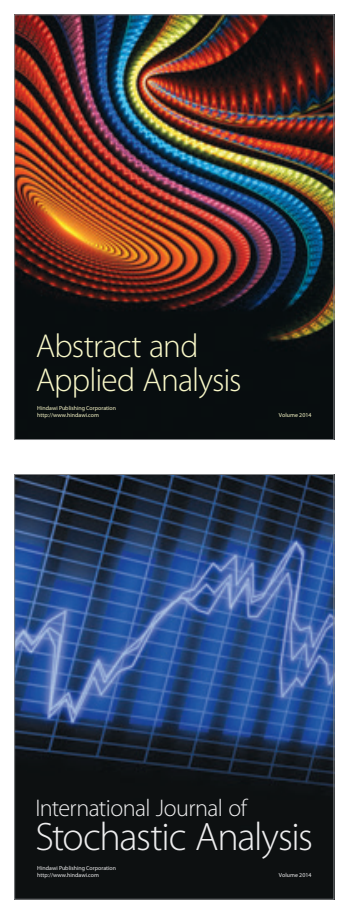

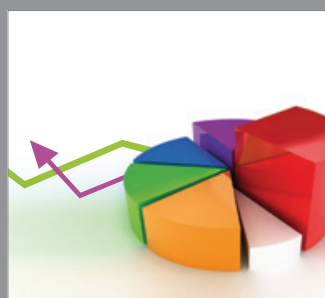

ournal of

Probability and Statistics

Promensencen
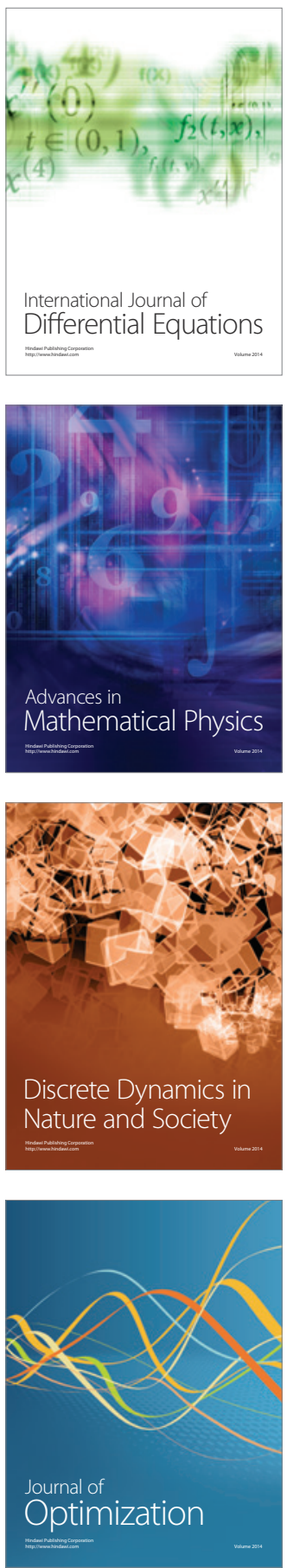